\title{
Corrigendum: Release of SOS2 kinase from sequestration with GIGANTEA determines salt tolerance in Arabidopsis
}

Woe-Yeon Kim, Zahir Ali, Hee Jin Park, Su Jung Park, Joon-Yung Cha, Javier Perez-Hormaeche, Francisco Javier Quintero, Gilok Shin, Mi Ri Kim, Zhang Qiang, Li Ning, Hyeong Cheol Park, Sang Yeol Lee, Ray A. Bressan, Jose M. Pardo, Hans J. Bohnert \& Dae-Jin Yun

Nature Communications 4:1352 doi:10.1038/ncomms2357 (2013); Published 15 Jan 2013; Updated 7 May 2013

An image in Fig. 5a in this Article, showing CBB-stained bands for the 'Phosphatase' group, was inadvertently duplicated from the 'Control' group. The correct version of the figure appears below.

a

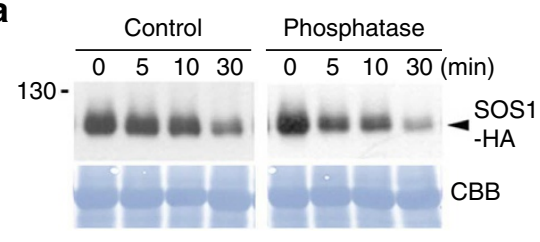

C

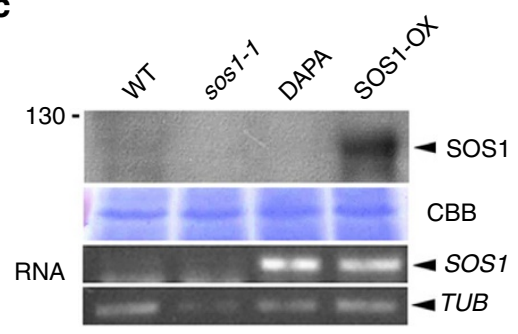

e

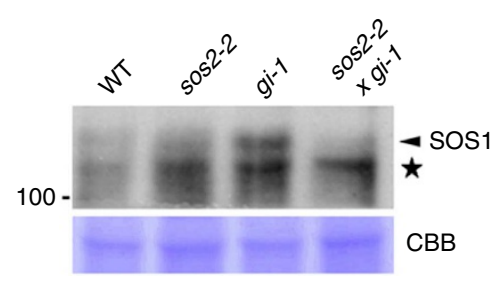

b

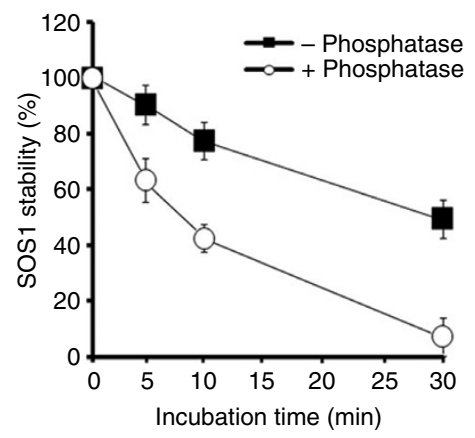

d

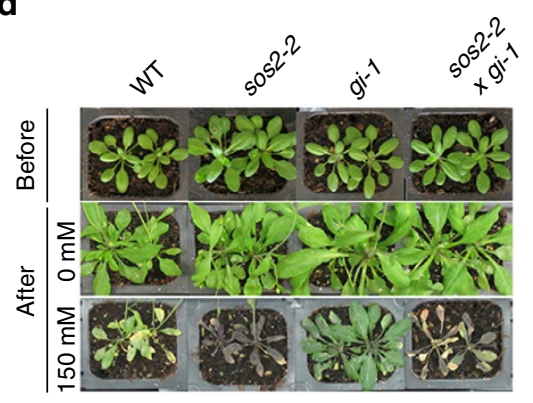

